\title{
Ultrafast Coherent Control of Spin Precession Motion by Terahertz Magnetic Pulses
}

\author{
M. Nakajima*, K. Yamaguchi and T. Suemoto \\ Institute for Solid State Physics, The University of Tokyo, Kashiwanoha 5-1-5, Kashiwashi, Chiba, 277-8581, Japan \\ We demonstrated coherent control of spin precession motion due to the ferromagnetic resonance induced by \\ magnetic field component of ultrashort terahertz pulses. The amplitude of the precession can be controlled by \\ the pulse separation time of the double pulse excitation technique. We succeeded in observing the energy transfer \\ between spin and photon systems, and the energy of the spin system is returned to the second terahertz pulses \\ instantaneously when the precession amplitude is cancelled.
}

PACS: 75.78.Jp, 42.50.Md, 76.50.+g, 78.20.Ls

\section{Introduction}

Ultrafast optical coherent control of the spin degrees of freedom is currently receiving intense attention [1-9]. Preceding reports on demonstrations of coherently controlling the spin systems have been conducted using indirect excitations of the spins that occur as a result of dielectric interaction with optical pulses such as electronic excitations and inverse Faraday effect [3-9]. Detecting methods of the spin precessions in these studies are generally also indirect and monitor the spins through fluctuation of dielectric constants. Very recently, we succeeded to show that spin precessions are directly excited by magnetic field of a terahertz pulse and observed in time domain via terahertz $(\mathrm{THz})$ emission with a circular polarization from the spin system [1]. Kampfrath et al. reported the coherent control of an antiferromagnetic spin wave by using $\mathrm{THz}$ magnetic pulse excitations and optical Faraday detection techniques in antiferromanget $\mathrm{NiO}[9]$. However, there is no report of coherent control on the ferromagnetic spin wave, and the ferromagnetic spin response is important for the magneto-optic devices. In this report, we will demonstrate coherent control of spin precession motions on a macroscopic magnetization in canted antiferromagnet $\mathrm{YFeO}_{3}$ by detecting circular polarized $\mathrm{THz}$ emission due to spin precession controlled by the double pulse excitation technique using impulsive $\mathrm{THz}$ magnetic fields [2].

The concept of our experiment is shown in Fig. 1. The impulsive magnetic field associated with an ultrashort $\mathrm{THz}$ pulse $\left(\boldsymbol{H}_{\mathrm{THz}}\right)$ tilts the magnetic moment $(\boldsymbol{M})$ as shown by this equation

$$
\frac{\mathrm{d} \boldsymbol{M}}{\mathrm{d} t} \propto \boldsymbol{M} \times \boldsymbol{H},
$$

* corresponding author; e-mail: nak@issp.u-tokyo.ac.jp (a)

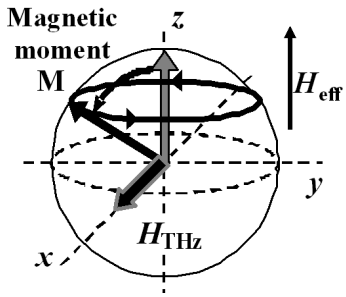

(b)

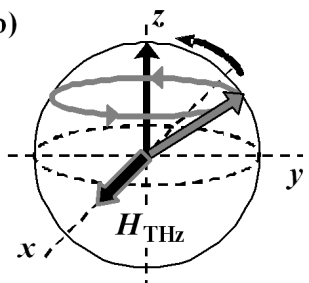

Fig. 1. Illustration showing the dynamics of magnetic moment. (a) The magnetic moment $(\boldsymbol{M})$ is tilted within $y-z$ plane by the impulsive magnetic field by the first pulse $\left(\boldsymbol{H}_{\mathrm{THz}}\right)$ along $x$-axis, and begins precession motion around $z$-axis $\left(\| \boldsymbol{H}_{\text {eff }}\right)$. (b) Inserting the second magnetic pulse at a specific time, the precession of the magnetic moment stops and the moment is directed along $z$-axis.

where $\boldsymbol{H}$ indicates the sum of the internal effective magnetic field $\left(\boldsymbol{H}_{\mathrm{eff}}\right)$ and $\boldsymbol{H}_{\mathrm{THz}}$. The magnitude of $\boldsymbol{H}_{\mathrm{THz}}$ is much smaller than that of $\boldsymbol{H}_{\text {eff }}$. After this tipping of $\boldsymbol{M}$ by $\boldsymbol{H}_{\mathrm{THz}}, \boldsymbol{M}$ starts to precess around $\boldsymbol{H}_{\mathrm{eff}}$ along the $z$-axis direction (Fig. 1a). The rotating coherent bulk magnetization radiates circularly polarized electromagnetic wave emission at a frequency of ferromagnetic resonance. This phenomenon is so-called free induction decay (FID) of the spin system in ferromagnet. Furthermore, inserting the second magnetic pulse with the appropriate time interval as shown in Fig. 1b, the rotated magnetic moment stops and the moment is directed along $z$-axis. The magnetic component of the $\mathrm{THz}$ pulse with a half-cycled wave form can be regarded as an ultrashort magnetic pulse with the pulse duration of $\approx 0.3 \mathrm{ps}$. We can probe directly the motion of the magnetic moment by observing the $\mathrm{THz}$ emission from the magnetic moment.

\section{Experiment}

Transmission-type THz time-domain spectroscopy [1] was performed at room temperature. A mode-locked 
Ti:sapphire laser delivered ultrashort (12 fs) light pulses with a central photon energy of $1.55 \mathrm{eV}$ at a repetition rate of $75 \mathrm{MHz}$. The emitter and detector of the $\mathrm{THz}$ waves were low-temperature-grown GaAs photoconductive antennas. The emitted $\mathrm{THz}$ wave was collimated and incident on the sample. As it will be seen in the following part (Fig. 2), the incident $\mathrm{THz}$ wave is close to monocycle with positive and negative going amplitudes. However, the time width (full width at half maximum) of the negative component around $-2 \mathrm{ps}$ is $2.1 \mathrm{ps}$, which is longer than the half-period of the spin precession (1.67 ps). The slowly varying magnetic field slightly tilts the rotation axis and does not excite the precession motion. Thus the main effect of this incident $\mathrm{THz}$ pulse on the precession motion comes from the positive going component, which is narrower than the half-period. Therefore, we interpret that the $\mathrm{THz}$ wave works effectively on spin systems as a "half cycle" pulse. The transmitted THz field was detected by the photoconductive antenna, which was triggered by optical probe pulses with a variable time delay. The incident $\mathrm{THz}$ waves have horizontally polarized electric field and vertically polarized magnetic field. The horizontal and vertical electric field was measured by using the free standing wire-grid polarizers [1]. Excitation pulses were doubled by the Michelson-type interferometer or hemispherical mirrors [2] with a different pulse separation time $\Delta t$.

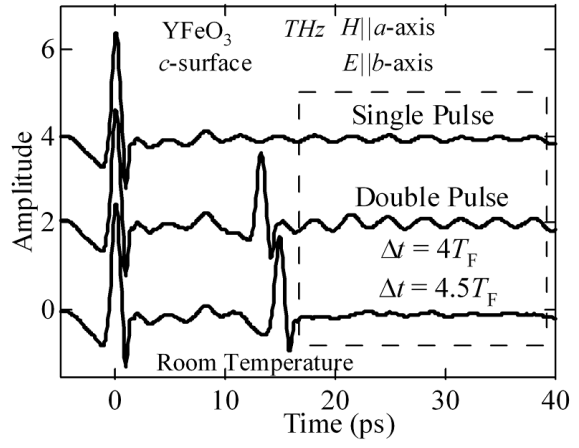

Fig. 2. Temporal wave forms of transmitted $\mathrm{THz}$ wave through $c$-cut plane parallel plate of $\mathrm{YFeO}_{3}$ for single and double pulse excitation. The wave forms consist of the half-cycle $\mathrm{THz}$ main pulse and oscillation components which reflect the spin precession motion.

The samples used in this study are single crystals of yttrium orthoferrite $\mathrm{YFeO}_{3}$ grown by the floating zone method. $\mathrm{YFeO}_{3}$ is an antiferromagnet below the Néel temperature $T_{\mathrm{N}}=645 \mathrm{~K}[10]$, and nearest neighbors of $\mathrm{Fe}^{3+}$ ions are ordered in antiparallel alignment along the $a$-axis of the orthorhombic crystal lattice [11]. $\mathrm{YFeO}_{3}$ possesses macroscopic magnetization in the direction of the $c$-axis ascribed to canting of $\mathrm{Fe}^{3+}$ ion magnetic moments, and have two branches of antiferromagnetic resonance in sub- $\mathrm{THz}$ frequency range. One is called quasi-antiferromagnetic mode (AF-mode) and has resonant frequency of $0.527 \mathrm{THz}$ at $300 \mathrm{~K}$. The other branch with its resonant frequency at $0.299 \mathrm{THz}(300 \mathrm{~K})$ is called quasi-ferromagnetic mode (F-mode), which is interpreted as a precession motion of the macroscopic magnetization [12]. In this study, a $c$-surface platelet with a thickness of $1.4 \mathrm{~mm}$ was prepared. A static magnetic field of $\approx 0.1 \mathrm{~T}$ was applied along the $c$-axis (the Faraday layout) to remove the quasi-ferromagnetic domain structure in the measurement. The polarizations of the magnetic and electric fields of input $\mathrm{THz}$ pulses are parallel to $a$-axis and $b$-axis, respectively. In this polarization layout, the $x-, y$-, $z$-axes in Fig. 1 correspond to $a-, b$-, $c$-axes of crystal structure in $\mathrm{YFeO}_{3}$, respectively.

\section{Results and discussion}

Figure 2 shows the experimental result of the $\mathrm{THz}$ wave forms transmitted through the sample with single and double pulse excitations. The signals show the horizontally polarized component of the electric field $(E \| b$ -axis) which is proportional to the vertically polarized component of the magnetic field $\left(H_{\mathrm{THz}} \| a\right.$-axis $)$. The wave forms consist of the "half-cycle" $\mathrm{THz}$ main pulse and oscillation components by the spin precessions due to the ferromagnetic resonance (the oscillation period is $T_{\mathrm{F}} \approx 3.3 \mathrm{ps}$ or the frequency is $0.299 \mathrm{THz}$ ). The oscillation decays with a time constant of $\approx 40 \mathrm{ps}$ as a consequence of the FID radiation and other relaxation processes such as spin-phonon and spin-spin relaxations within the condensed matter. The oscillation amplitude after the second main pulse for double pulse excitation is enhanced at $\Delta t=4 T_{\mathrm{F}}$. On the other hand, the oscillation amplitude for $\Delta t=4.5 T_{\mathrm{F}}$ are cancelled. These facts indicate that we succeeded in the coherent control of the spin precession by the ultrashort magnetic pulses and the amplitude can be controlled by the time interval of the $\mathrm{THz}$ magnetic pulses.

Figure 3 shows the three-dimensional trajectory plots of the oscillation part of the horizontal ( $E \| b$-axis) and vertical ( $E \| a$-axis) polarization components, and obviously demonstrates circular polarization which reflects FID signal of the spin precession motion induced by the $\mathrm{THz}$ magnetic pulses. The results of the double pulse excitation at $\Delta t=4 T_{\mathrm{F}}$ and $\Delta t=4.5 T_{\mathrm{F}}$ indicate that the amplitude of spin precession motion changes drastically by changing the pulse separation time. It is confirmed that the amplitude of the spin precession is damped for $\Delta t=4.5 T_{\mathrm{F}}$ (Fig. 3c).

We will discuss an energy transfer in coherent control as follows. In the case of $\Delta t=4 T_{\mathrm{F}}$, the amplitude is increased twice as much as that in single pulse excitation as shown in Fig. 2, i.e., the stored energy of the spin precession, which is proportional to square of the amplitude, is four times larger compared to that in the single pulse excitation case. On the other hand, the amplitude for $\Delta t=4.5 T_{\mathrm{F}}$ becomes zero and the energy of the spin system also becomes zero. In order to see the destination of the energy transferred from the spin system, we highlight the main peak of the second $\mathrm{THz}$ pulse 


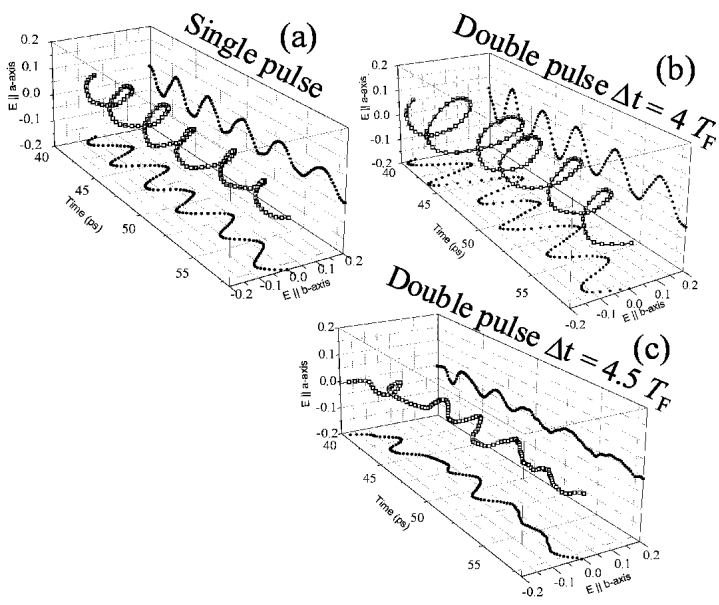

Fig. 3. Three-dimensional trajectory plots for the oscillation components after the main pulse of the transmitted $\mathrm{THz}$ electric fields with both horizontal $(E \| b$ -axis) and vertical ( $E \| a$-axis) components. Part (a) shows the plot at single pulse excitation. (b), (c) show the plot at the double pulse excitation with $\Delta t=4 T_{\mathrm{F}}$ and $4.5 T_{\mathrm{F}}$, respectively.

as shown in Fig. 4. In the case of $\Delta t=4 T_{\mathrm{F}}$, the peak amplitude of main pulse is lower than that of the single pulse excitation (without the first pulse), then the part of the energy in $\mathrm{THz}$ pulse moves to the spin system. On the other hand, the peak amplitude of the main pulse for $\Delta t=4.5 T_{\mathrm{F}}$ is larger than that of single pulse excitation. This result indicates that the stored energy in spin system excited by the first pulse has been transferred to $\mathrm{THz}$ pulse instantaneously.

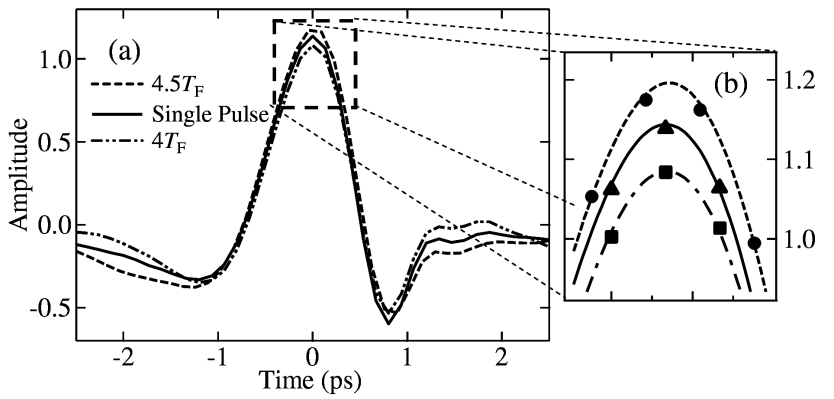

Fig. 4. (a) Main peaks of the second pulses in horizontal $(E \| b)$ polarization under single pulse excitation (second pulse without a first pulse, solid curve), $\Delta t=4 T_{\mathrm{F}}$ (chain curve) and $4.5 T_{\mathrm{F}}$ (dashed curve) condition. (b) The enlargement around the peak of the second pulse.

We estimated roughly the energy exchanged between spin and photon systems. The energy of the electromagnetic wave is estimated by calculating time integration of the square of the amplitude. The energy difference in the main peak between at $\Delta t=4 T_{\mathrm{F}}$ and $4.5 T_{\mathrm{F}}$ and the total energy of the enhanced FID at $\Delta t=4 T_{\mathrm{F}}$ are compared. Although the difference of the peak height of the main pulse of the second pulse is seen small in the scale of amplitude as shown in Fig. 4, the energy difference in the main peak becomes comparable to the total energy of the enhanced FID as follows.

The height at $4 T_{\mathrm{F}}$ and $4.5 T_{\mathrm{F}}$ in amplitude are about 1.08 and 1.2 on the scale of Fig. 4a, respectively. By squaring these and multiplying by full width at half maximum (FWHM) of the peak which slightly differs in $4 T_{\mathrm{F}}$ and $4.5 T_{\mathrm{F}}, 0.56$ and 0.59 ps respectively, we obtain total energy of the main peaks 0.653 for $4 T_{\mathrm{F}}$ and 0.850 for $4.5 T_{\mathrm{F}}$. Therefore, difference in the total energy of the main peak is " $0.197 . "$

Next, the total energy of the FID at $4 T_{\mathrm{F}}$ just after the second pulse is estimated from Fig. 3b. The amplitude of the oscillation in horizontal polarization is about 0.1 . Because FWHM of one cycle in square of a sinusoid is $1 / 4$ of the oscillating period, square of a sinusoid with oscillation period of $3.3 \mathrm{ps}$ has a peak with $0.825 \mathrm{ps}$ width for every 1.65 ps. This damped oscillation amplitude has a time constant of $\approx 40 \mathrm{ps}$, so the time constant in intensity is $20 \mathrm{ps}$ and there are about $20 \mathrm{ps} / 1.65 \mathrm{ps}=12.12$ peaks within this time constant. Therefore calculation of the total energy of the oscillating component in horizontal polarization yields 0.10 . In the vertical polarization the amplitude of oscillation is about $10 \%$ smaller than that in horizontal polarization, and the total energy in this polarization is 0.08 . Thus the overall energy of the FID emission is approximately " 0.18 ." These two values of " 0.198 " and " 0.18 " are very close and the change in total energy of the main peak is comparable with the difference in the total energy of the FID radiation after the second excitation.

It is confirmed that the transferred energy to/from the spin system can be explained by the energy change of the $\mathrm{THz}$ main pulse. The small difference of the values is ascribed to the energy dissipation such as spin-spin and spin-phonon relaxations within sample. This fact indicates that the energy exchanged between spin and photon system is performed practically losslessly, and this can be regarded as the demonstration of "read out" the spin state by using the $\mathrm{THz}$ pulses. This technique of the coherent control using $\mathrm{THz}$ magnetic pulse is very useful for the applications such as spintronics and quantum information because we can write and read out information in a spin system in ultrafast time scale.

Furthermore, this $\mathrm{THz}$ coherent control technique has an advantage compared to the coherent control using optical pulses. The $\mathrm{THz}$ pulses can excite and detect only spin system without other excitation such as the electronic excitation because the $\mathrm{THz}$ photon energy is very small. The energy efficiency of the coherent control using $\mathrm{THz}$ pulses is very high and this technique enables us to control the spin system with several orders lower energy compared to that using optical pulses.

\section{Conclusion}

We demonstrated the ultrafast coherent control of spin precession motion by the impulsive magnetic field us- 
ing the double pulse technique. The amplitude of the spin precession motion is enhanced twice at $\Delta t \sim 4 T_{\mathrm{F}}$, whereas it becomes zero at $\Delta t \sim 4.5 T_{\mathrm{F}}$.

We succeeded in observing directly the energy transfer between spin and photon spin systems, and the energy of the spin system is returned to the second pulse instantaneously at the case of the cancellation. This new technique using ultrashort magnetic pulses opens up the possibility of new application for the spin control in the field of quantum information and spintronics, because the effective usage of $\mathrm{THz}$ magnetic pulses enables us to observe directly the response of the magnetic dipole transition.

\section{Acknowledgments}

This work has been supported in part by a Grant-in-Aid for Young Scientists (A) (No. 23686012) from JSPS. K.Y. is grateful to JSPS Research Fellowships for Young Scientists.

\section{References}

[1] M. Nakajima, A. Namai, S. Ohkoshi, T. Suemoto, Opt. Exp. 18, 18260 (2010).

[2] K. Yamaguchi, M. Nakajima, T. Suemoto, Phys. Rev. Lett. 105, 237201 (2010).
[3] A.V. Kimel, A. Kirilyuk, P.A. Usachev, R.V. Pisarev, A.M. Balbashov, T. Rasing, Nature 435, 655 (2005).

[4] R. Akimoto, K. Ando, F. Sasaki, S. Kobayashi, T. Tani, J. Appl. Phys. 84, 6318 (1998).

[5] J.A. Gupta, R. Knobel, N. Samarth, D.D. Awschalom, Science 292, 2458 (2001).

[6] T. Satoh, N.P. Duong, M. Fiebig, Phys. Rev. B 74, 012404 (2006).

[7] A.V. Kimel, A. Kirilyuk, T. Rasing, Laser Photon. Rev. 1, 275 (2007).

[8] E. Rozkotová, P. Němec, N. Tesarová, P. Malý, V. Novák, K. Olejník, M. Cukr, T. Jungwirth, Appl. Phys. Lett. 93, 232505 (2008).

[9] T. Kampfrath, A. Sell, G. Klatt, A. Pashkin, S. Mahrlein, T. Dekorsy, M. Wolf, M. Fiebig, A. Leitenstorfer, R. Huber, Nature Photon. 5, 31 (2011).

[10] K.N. Kocharyan, R.M. Martirosyan, V.G. Prpryan, E.L. Sarkisyan, Sov. Phys. JETP 59, 373 (1984).

[11] H. Lüttgemeir, H.G. Bohn, M. Brajczewska, J. Magn. Magn. Mater. 21, 289 (1980).

[12] G.V. Kozlov, S.P. Lebedev, A.A. Mukhin, A.S. Prokhorov, I.V. Fedorov, A.M. Balbashov, I.Y. Parsegov, IEEE Trans. Magn. 29, 3443 (1993). 